Research Article

\title{
Computing the Hosoya Polynomial of $M$-th Level Wheel and Its Subdivision Graph
}

\author{
Peng Xu (D), ${ }^{1,2}$ Muhammad Numan, ${ }^{3}$ Aamra Nawaz, ${ }^{3}$ Saad Ihsan Butt $\mathbb{D}^{\circ},{ }^{4}$ Adnan Aslam $\left(\mathbb{D},{ }^{5}\right.$ \\ and Asfand Fahad $\mathbb{D i D}^{6}$ \\ ${ }^{1}$ Institute of Computational Science and Technology, Guangzhou University, Guangzhou, China \\ ${ }^{2}$ School of Computer Science of Information Technology, Qiannan Normal University for Nationalities, Duyun 558000, China \\ ${ }^{3}$ Department of Mathematics, COMSATS University Islamabad, Attock Campus, Islamabad, Pakistan \\ ${ }^{4}$ Department of Mathematics, COMSATS University Islamabad, Lahore Campus, Islamabad, Pakistan \\ ${ }^{5}$ Department of Natural Sciences and Humanities, University of Engineering and Technology, Lahore (RCET), Lahore, Pakistan \\ ${ }^{6}$ Department of Mathematics, COMSATS University Islamabad, Vehari 61100, Pakistan
}

Correspondence should be addressed to Asfand Fahad; asfandfahad1@yahoo.com

Received 3 June 2021; Accepted 23 October 2021; Published 27 November 2021

Academic Editor: Ajaya Kumar Singh

Copyright ( 92021 Peng Xu et al. This is an open access article distributed under the Creative Commons Attribution License, which permits unrestricted use, distribution, and reproduction in any medium, provided the original work is properly cited.

The determination of Hosoya polynomial is the latest scheme, and it provides an excellent and superior role in finding the Weiner and hyper-Wiener index. The application of Weiner index ranges from the introduction of the concept of information theoretic analogues of topological indices to the use as major tool in crystal and polymer studies. In this paper, we will compute the Hosoya polynomial for multiwheel graph and uniform subdivision of multiwheel graph. Furthermore, we will derive two well-known topological indices for the abovementioned graphs, first Weiner index, and second hyper-Wiener index.

\section{Introduction}

Let $G$ be a finite connected graph with vertex set $V(G)=V$ and edge set $E(G)=E$. The distance $d_{u, v}$ between $u, v \in V(G)$ is the length of the shortest path joining $u, v$. The diameter $d(G)$ of $G$ is $\max _{(u, v)} d_{u, v}$. The terminologies not defined here can be seen in [1,2]. The Weiner index $W$ was first put forward in chemistry by Harold Weiner to compute the cardinality of the carbon-carbon bonds among all pairs of carbon atoms in alkane. For a molecular graph $G$, it is defined as

$$
W(G)=\sum_{u, v \in V} d_{u, v}
$$

To read more about the chemical application of Weiner index, see [3-6], and for its mathematical properties, see $[7,8]$.

Milan Randic coined the term hyper-Wiener index WW $(G)$ of $G[9]$ as

$$
\mathrm{WW}(G)=\frac{1}{2} \sum_{u, v \in V}\left(d_{u, v}+d_{u, v}^{2}\right)
$$

To read more the properties of hyper-Weiner index, see [9-12]. Hosoya polynomial was first introduced by Hosoya [13] and it received the attention of a lot of researchers. The same notion was independently put forward by Sagan et al. [14] as Weiner polynomial $G$. The Hosoya polynomial $H(G, x)$ of $G$ is defined as

$$
H(G, x)=\sum_{u, v \in V}\left(x^{d_{u, v}}\right) .
$$

Let $\alpha(G, k)$ be the number of ordered pair $(u, v)$ in $V$ with $d_{u, v}=k$. Then, the above definition of Hosoya polynomial can be expressed as

$$
H(G, x)=\sum_{k=0}^{d(G)}\left(\alpha(G, k) x^{k}\right.
$$


The Hosoya polynomial has been investigated on polycyclic aromatic hydrocarbons [15], benzenoid chains [16], Fibonacci and Lucas cubes [17], zigzag polyhexnanotorus [9], carbon nanotubes [18], Hanoi graphs [19], and circumcoronene series [20]. A significant importance of $H(G, x)$ is that some distance-based topological indices (TIs) such as $W(G)$ and WW $(G)$ of $G$ can be computed from the Hosoya polynomial as

$$
W(G)=H^{\prime}(G: 1), W W(G)=H^{\prime}(G ; 1)+\frac{1}{2} H^{\prime \prime}(G ; 1) .
$$

The readers can see the following papers [21-25] for the results on distance-based TIs.

\section{Hosoya Polynomial of $M$-th Level Wheel Graph}

For $n \geq 2$, the join $K_{1} \vee C_{n}$ is called a wheel graph denoted by $W_{n+1}$. The vertex that comes from the graph $K_{1}$ is called the core and is denoted by $c$. It has order $n+1$ and size $2 n$. A $m$-level wheel graph denoted by $m W_{n}$ is the graph obtained by taking $m$ copies of the cycle $C_{n}$ and one copy of $K_{1}$, such that all the vertices of each copy of $C_{n}$ are adjacent with the core vertex $c$. The graph of $m W_{n}$ is depicted in Figure 1. Note that $m W_{n}$ has $m n+1$ vertices and $2 m n$ edges. If we label the vertices of cycle at the $m$-th level by $u_{0}^{m}, u_{1}^{m}, u_{2}^{m}, \ldots, u_{n-1}^{m}$, then the $V\left(m W_{n}\right)$ and the $E\left(m W_{n}\right)$ can be written as

$$
\begin{aligned}
& V\left(m W_{n}\right)=\left\{c, u_{i}^{j}, 0 \leq i \leq n-1,1 \leq j \leq m\right\}, \\
& E\left(m W_{n}\right)=\left\{c u_{i}^{j}, 0 \leq i \leq n-1,1 \leq j \leq m\right\} \cup\left\{u_{i}^{j} u_{i+1}^{j}, 0 \leq i \leq n-1,1 \leq j \leq m\right\} .
\end{aligned}
$$

Next, the theorem gives the expression for the Hosoya polynomial of $m W_{n}$.

Theorem 1. Let $m, n \geq 1$, then $H\left(m W_{n} ; x\right)$ is of the form

$$
\begin{aligned}
H\left(m W_{n} ; x\right)= & (m n+1)+(2 m n) x \\
& +\left[m n(n-3)+\frac{(n(m-1))^{2}(m)}{2}\right] x^{2} .
\end{aligned}
$$

Proof. It is easy to observe that the diameter of $m W_{n}$ is 2 . In order to derive the $H\left(m W_{n} ; x\right)$, we compute the coefficients $\alpha\left(m W_{n}, k\right)$ for $k=0,1,2$. By definition, we have $\alpha\left(m W_{n}, 0\right)=m n+1$ and $\alpha\left(m W_{n}, 1\right)=2 m n$. To compute $\alpha\left(m W_{n}, 2\right)$, we use the following notation:

$$
\alpha_{A}=\text { number of pair of vertices in set } A \text {. }
$$

The cardinality of order pairs in $V\left(m W_{n}\right)$ with distance 2 can be characterized by the following two sets:

$$
\begin{aligned}
& A_{1}=\left\{\left(u_{i}^{j}, u_{i+l}^{j}\right), \quad 1 \leq j \leq m, 0 \leq i \leq n-1,2 \leq l \leq n-2\right\}, \\
& A_{2}=\left\{\left(u_{i}^{j}, u_{l}^{h}\right), \quad 0 \leq i \leq n-1,0 \leq l \leq n-1,1 \leq j \leq m-1, j+1 \leq h \leq m\right\} .
\end{aligned}
$$

The cardinality of the above sets is $\alpha_{A_{1}}=m n(n-3)$ and $\alpha_{A_{2}}=(n(m-1))^{2}(m) / 2$ and hence the coefficient $\alpha\left(m W_{n}, 2\right)$ is equal to $\alpha\left(m W_{n}, 2\right)=\alpha_{A_{1}}+\alpha_{A_{2}}=m n(n-3)+$ $(n(m-1))^{2}(m) / 2$. Now, using the values of $\alpha\left(m W_{n}, 0\right), \alpha\left(m W_{n}, 1\right)$, and $\alpha\left(m W_{n}, 2\right)$, we get the desired result. 


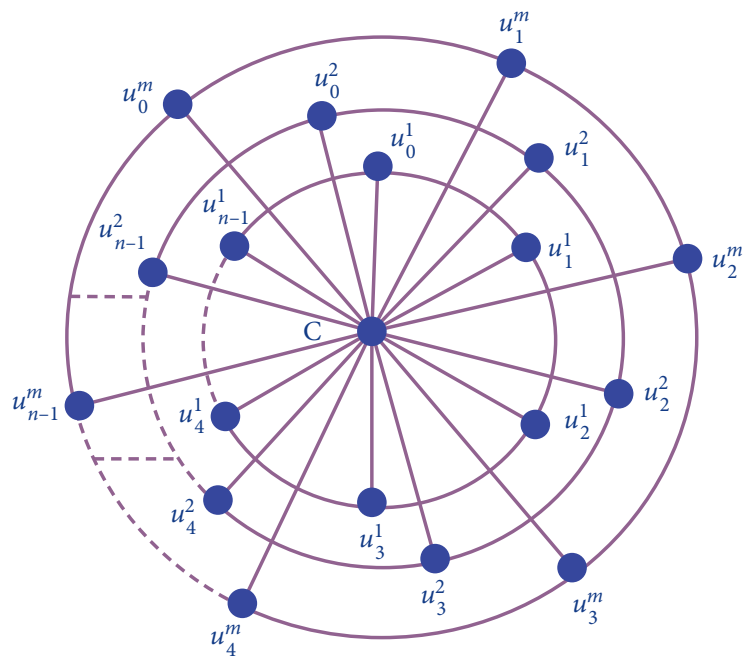

Figure 1: Multiwheel graph $m W_{n}$.

Corollary 1. Let $m, n \geq 1$, then $W\left(m W_{n}\right)$ and $W W\left(m W_{n}\right)$ are given as

$$
\begin{aligned}
W\left(m W_{n}\right) & =(2 m n)+2\left[m n(n-3)+\frac{(n(m-1))^{2}(m)}{2}\right], \\
W W\left(m W_{n}\right) & =(2 m n)+3\left[m n(n-3)+\frac{(n(m-1))^{2}(m)}{2}\right] .
\end{aligned}
$$

\section{Hosoya Polynomial of Subdivision of $M$-th Level Wheel Graph}

The subdivision graph $S\left(m W_{n}\right)$ of $m W_{n}$ is constructed from $m W_{n}$ by adding a vertex into each edge of $m W_{n}$. In other words, we replace each edge of $m W_{n}$ by a path of length 2 . The graph of $S\left(m W_{n}\right)$ is depicted in Figure 2. If we label the new vertices that we insert in the cycle at the $j$-th level by $x_{1}^{j}, x_{2}^{j}, \ldots, x_{n-1}^{j}$ for $j=1,2, \ldots, m$, then the vertex set and edge set of $S\left(m W_{n}\right)$ can be written as

$$
\begin{aligned}
& V\left(S\left(m W_{n}\right)\right)=\left\{c, u_{i}^{j}, v_{i}^{j}, x_{i}^{j}, \quad 0 \leq i \leq n-1,1 \leq j \leq m\right\}, \\
& E\left(S\left(m W_{n}\right)\right)=\left\{c v_{i}^{j}, v_{i}^{j} u_{i}^{j}, x_{i}^{j} u_{i}^{j}, u_{i}^{j} x_{i+1}^{j}, \quad 0 \leq i \leq n-1,1 \leq j \leq m\right\} \cup\left\{u_{i}^{j} u_{i+1}^{j}, \quad 0 \leq i \leq n-1,1 \leq j \leq m\right\} .
\end{aligned}
$$

It is easy to observe that order and size of are $3 m n+1$ and $4 m n$, respectively. In the next theorem, we give the analytic formula to derive the $H\left(S\left(m W_{n}\right) ; x\right)$.
Theorem 2. Let $m, n \geq 1$, then the $H\left(S\left(m W_{n}\right) ; x\right)$ is of the form

$$
\begin{aligned}
H\left(S\left(m W_{n}\right) ; x\right)= & (3 m n+1)+(4 m n) x+[5 m n+m n(n-1)] x^{2} \\
& +\left[\frac{(m n)^{2}(m-1)}{2}+3 m n+m n(n-1)\right] x^{3}+\left[m((m-1) n)^{2}+m n(2 n-5)+m n\right] x^{4} \\
& +\left[m n(n-4)+\frac{m((m-1) n)^{2}}{2}\right] x^{5}+\left[m n(n-5)+\frac{m((m-1) n)^{2}}{2}\right] x^{6} .
\end{aligned}
$$

Proof. It is easy to observe that the diameter of $m W_{n}$ is 6. In order to derive the $H\left(S\left(m W_{n}\right) ; x\right)$, we find the coefficients $\alpha\left(S\left(m W_{n}\right), k\right)$ for $k=0,1,2, \ldots, 6$. By definition, we have $\alpha\left(S\left(m W_{n}\right), 0\right)=3 m n+1$ and $\alpha\left(S\left(m W_{n}\right), 1\right)=4 m n$. To compute $\alpha\left(S\left(m W_{n}\right), j\right)$ for $j=2,3,4,5,6$, we use the following notation:

$$
\alpha_{A}=\text { number of pair of vertices in set } A .
$$




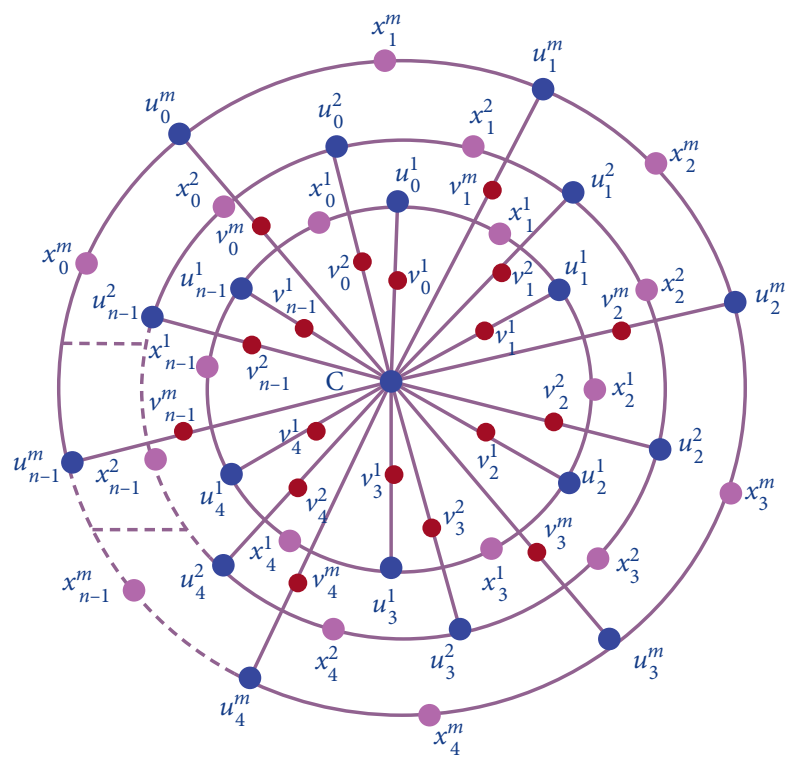

FIGURE 2: Uniform subdivision of multiwheel graph $m S_{n}$.

The cardinality of order pairs in $V\left(S\left(m W_{n}\right)\right)$ at distance 2 can be characterized by the following sets:

$$
\begin{array}{ll}
B_{1}=\left\{\left(c, u_{i}^{j}\right),\right. & 1 \leq j \leq m, 0 \leq i \leq n-1\}, \\
B_{2}=\left\{\left(v_{i}^{j}, v_{i+l}^{j}\right),\right. & 1 \leq j \leq m, 0 \leq i \leq n-1,1 \leq l \leq n-1\}, \\
B_{3}=\left\{\left(v_{i}^{j}, x_{i+1}^{j}\right),\right. & 1 \leq j \leq m, 0 \leq i \leq n-1\}, \\
B_{4}=\left\{\left(v_{i}^{j}, x_{i}^{j}\right),\right. & 0 \leq i \leq n-1,1 \leq j \leq m\}, \\
B_{5}=\left\{\left(u_{i}^{j}, u_{i+1}^{j}\right),\right. & 1 \leq j \leq m, 0 \leq i \leq n-1\}, \\
B_{6}=\left\{\left(x_{i}^{j}, x_{i+1}^{j}\right),\right. & 1 \leq j \leq m, 0 \leq i \leq n-1\} .
\end{array}
$$

The cardinality of the above sets is $\alpha_{B_{1}}=\alpha_{B_{3}}=\alpha_{B_{4}}=\alpha_{B_{5}}=\alpha_{B_{6}}=m n, \quad \alpha_{B_{2}}=m n(n-1), \quad$ and hence

$$
\begin{aligned}
\alpha\left(S\left(m W_{n}\right), 2\right) & =\alpha_{B_{1}}+\alpha_{B_{2}}+\alpha_{B_{3}}+\alpha_{B_{4}}+\alpha_{B_{5}}+\alpha_{B_{6}} \\
& =5 m n+m n(n-1) .
\end{aligned}
$$

The cardinality of order pairs in $V\left(S\left(m W_{n}\right)\right)$ at distance 3 can be characterized by the following sets:

$$
\begin{array}{ll}
C_{1}=\left\{\left(c, x_{i}^{j}\right),\right. & 1 \leq j \leq m, 0 \leq i \leq n-1\}, \\
C_{2}=\left\{\left(v_{i}^{j}, u_{i+l}^{j}\right),\right. & 1 \leq j \leq m, 0 \leq i \leq n-1,1 \leq l \leq n-1\}, \\
C_{3}=\left\{\left(v_{i}^{j}, u_{1}^{j+k}\right),\right. & 1 \leq j \leq m, 1 \leq k \leq m-j, 0 \leq i \leq n-1,0 \leq l \leq n-1\}, \\
C_{4}=\left\{\left(u_{i}^{j}, x_{i+2}^{j}\right),\right. & 1 \leq j \leq m, 0 \leq i \leq n-1\}, \\
C_{5}=\left\{\left(x_{i}^{j}, u_{i+1}^{j}\right),\right. & 1 \leq j \leq m, 0 \leq i \leq n-1\} .
\end{array}
$$

The cardinality of the above sets is $\alpha_{C_{1}}=\alpha_{C_{4}}=\alpha_{C_{5}}=$ $m n, \alpha_{C_{2}}=m n(n-1), \alpha_{C_{3}}=(m n)^{2}(m-1) / 2$, and hence 


$$
\begin{aligned}
\alpha\left(S\left(m W_{n}\right), 3\right) & =\alpha_{C_{1}}+\alpha_{C_{2}}+\alpha_{C_{3}}+\alpha_{C_{4}}+\alpha_{C_{5}} \\
& =\frac{(m n)^{2}(m-1)}{2}+3 m n+m n(n-1) .
\end{aligned}
$$

The cardinality of order pairs in $V\left(S\left(m W_{n}\right)\right)$ at distance 4 can be characterized by the following sets:

$$
\begin{array}{ll}
D_{1}=\left\{\left(v_{i}^{j}, x_{i+l}^{j}\right),\right. & 1 \leq j \leq m, 0 \leq i \leq n-1,2 \leq l \leq n-1\}, \\
D_{2}=\left\{\left(v_{i}^{j}, x_{l}^{j+k}\right),\right. & 1 \leq j \leq m-1,1 \leq k \leq m-j, 0 \leq i \leq n-1,0 \leq l \leq n-1\}, \\
D_{3}=\left\{\left(u_{i}^{j}, u_{i+l}^{j}\right),\right. & 1 \leq j \leq m, 0 \leq i \leq n-1,2 \leq l \leq n-2\}, \\
D_{4}=\left\{\left(u_{i}^{j}, u_{i}^{j+k}\right),\right. & 1 \leq j \leq m-1,1 \leq k \leq m-j, 0 \leq i \leq n-1,0 \leq l \leq n-1\}, \\
D_{5}=\left\{\left(x_{i}^{j}, x_{i+2}^{j}\right),\right. & 1 \leq j \leq m, 0 \leq i \leq n-1\} .
\end{array}
$$

The cardinality of the above sets is $\alpha_{D_{1}}=m n(n-2), \alpha_{D_{2}}=m((m-1) n)^{2} / 2, \alpha_{D_{3}}=m n(n-3)$, $\alpha_{D_{4}}=m((m-1) n)^{2^{2}} / 2, \alpha_{D_{5}}=m n$, and hence

$$
\begin{aligned}
\alpha\left(S\left(m W_{n}\right), 4\right) & =\alpha_{D_{1}}+\alpha_{D_{2}}+\alpha_{D_{3}}+\alpha_{D_{4}}+\alpha_{D_{5}} \\
& =m((m-1) n)^{2}+m n(2 n-4) .
\end{aligned}
$$

$$
\begin{array}{ll}
E_{1}=\left\{\left(u_{i}^{j}, x_{i+l}^{j}\right),\right. & 1 \leq j \leq m, 0 \leq i \leq n-1,3 \leq l \leq n-2\}, \\
E_{2}=\left\{\left(u_{i}^{j}, x_{l}^{j+k}\right), \quad 1 \leq j \leq m-1,1 \leq k \leq m-j, 0 \leq i \leq n-1,0 \leq l \leq n-1\right\} .
\end{array}
$$

The cardinality of the above sets is $\alpha_{E_{1}}=m n(n-4), \alpha_{E_{2}}=m((m-1) n)^{2} / 2$, and hence

$$
\begin{aligned}
\alpha\left(S\left(m W_{n}\right), 5\right) & =\alpha_{E_{1}}+\alpha_{E_{2}} \\
& =m n(n-4)+\frac{m((m-1) n)^{2}}{2} .
\end{aligned}
$$

$$
\begin{array}{ll}
F_{1}=\left\{\left(x_{i}^{j}, x_{i+l}^{j}\right),\right. & 1 \leq j \leq m, 0 \leq i \leq n-1,3 \leq l \leq n-3\}, \\
F_{2}=\left\{\left(x_{i}^{j}, x_{l}^{j+k}\right),\right. & 1 \leq j \leq m-1,1 \leq k \leq m-j, 0 \leq i \leq n-1,0 \leq l \leq n-1\} .
\end{array}
$$

The cardinality of the above sets is $\alpha_{F_{1}}=m n(n-5), \alpha_{F_{2}}=m((m-1) n)^{2} / 2$, and hence

$$
\begin{aligned}
\alpha\left(S\left(m W_{n}\right), 6\right) & =\alpha_{F_{1}}+\alpha_{F_{2}} \\
& =m n(n-5)+\frac{m((m-1) n)^{2}}{2} .
\end{aligned}
$$

The cardinality of order pairs in $V\left(S\left(m W_{n}\right)\right)$ at distance 6 can be characterized by the following sets:

\section{5 can be characterized by the following sets:}

The cardinality of order pairs in $V\left(S\left(m W_{n}\right)\right)$ at distance 


$$
\begin{aligned}
W\left(m S_{n}\right)= & (4 m n)+2[5 m n+m n(n-1)]+3\left[\frac{(m n)^{2}(m-1)}{2}+3 m n+m n(n-1)\right] \\
& +4\left[m((m-1) n)^{2}+m n(2 n-5)+m n\right]+5\left[m n(n-4)+\frac{m((m-1) n)^{2}}{2}\right] \\
& +6\left[m n(n-5)+\frac{m((m-1) n)^{2}}{2}\right], \\
W W\left(m S_{n}\right)= & (4 m n)+3[5 m n+m n(n-1)]+6\left[\frac{(m n)^{2}(m-1)}{2}+3 m n+m n(n-1)\right] \\
& +10\left[m((m-1) n)^{2}+m n(2 n-5)+m n\right]+15\left[m n(n-4)+\frac{m((m-1) n)^{2}}{2}\right] \\
& +21\left[m n(n-5)+\frac{m((m-1) n)^{2}}{2}\right] .
\end{aligned}
$$

\section{Conclusion}

We examined the Hosoya polynomial and two vastly studied TIs $W(G)$ and WW $(G)$ for multiwheel graph $m W_{n}$ and subdivision of multiwheel graph $m S_{n}$.

\section{Data Availability}

No data were used for this study.

\section{Disclosure}

Mathematics subject classification: 05C09, 05C92, 92E10.

\section{Conflicts of Interest}

The authors hereby declare that there are no conflicts of interest regarding the publication of this paper.

\section{Acknowledgments}

This work was supported by the National Natural Science Foundation of China, No. 62002079.

\section{References}

[1] G. Chartrand, Introduction to Graph Theory, Tata McGrawHill Education, New York, NY, USA, 2006.

[2] R. Diestel, Graph Theory, Springer-Verlag, Berlin, Germany, 1997.

[3] Z. Mihalic and N. Trinajstic, "A graph theoretical approach to structure property relationships," Journal of Chemical Education, vol. 69, pp. 701-712, 1992.

[4] I. Gutman, Y. N. Yeh, S. L. Lee, and Y. L. Luo, "Some recent results in the theory of the wiener number," Indian Journal of Chemistry, vol. 32A, pp. 651-661, 1993.

[5] I. Gutman and J. H. Potgieter, "Wiener index and intermolecular forces," Journal of the Serbian Chemical Society, vol. 62, pp. 185-192, 1997.

[6] D. H. Rouvray, "The rich legacy of half a century of the wiener index," in Topology in Chemistry Discrete Mathematics of Molecules, D. H. Rouvray and R. B. King, Eds., Horwood, Chichester, UK, pp. 16-37, 2002.
[7] A. A. Dobrynin, R. Entringer, and I. Gutman, "Wiener index of trees: theory and applications," Acta Applicandae Mathematica, vol. 66, no. 3, pp. 211-249, 2001.

[8] A. A. Dobrynin, I. Gutman, S. Klavžar, and P. Žigert, "Wiener index of hexagonal systems," Acta Applicandae Mathematica, vol. 72, no. 3, pp. 247-294, 2002.

[9] M. Randic, "Novel molecular descriptor for structure property studies," Chemical Physics Letters, vol. 211, pp. 478-483, 1993.

[10] D. J. Klein, I. Lukovits, and I. Gutman, "On the definition of the hyper-wiener index for cycle-containing structures," Journal of Chemical Information and Computer Sciences, vol. 35, no. 1, pp. 50-52, 1995.

[11] I. Gutman, "Relation between hyper-wiener and wiener index," Chemical Physics Letters, vol. 364, no. 3-4, pp. 352-356, 2002.

[12] I. Gutman and B. Furtula, "Hyper-wiener index vs. wiener index. two highly correlated structure-descriptors," Monatshefte für Chemie-Chemical Monthly, vol. 134, no. 7, pp. 975-981, 2003.

[13] H. Hosoya, "On some counting polynomials in chemistry," Discrete Applied Mathematics, vol. 19, no. 1-3, pp. 239-257, 1988.

[14] B. E. Sagan, Y.-N. Yeh, and P. Zhang, "The wiener polynomial of a graph," International Journal of Quantum Chemistry, vol. 60, no. 5, pp. 959-969, 1996.

[15] S. Nikolic, N. Trinajstic, and Z. Mihalic, "The Wiener index: development and applications," Croatica Chemica Acta, vol. 68, pp. 105-129, 1995.

[16] W. Gao, Y. Wang, B. Jamil, and M. Kamran, "Characteristics studies of molecular structures in drugs," Saudi Pharmaceutical Journal, vol. 25, no. 4, pp. 580-586, 2017.

[17] N. PrabhakaraRao and A. LaxmiPrasanna, "On the Wiener index of pentachains," Applied Mathematical Sciences, vol. 2, pp. 2443-2457, 2008.

[18] A. A. Ali and A. M. Ali, "Hosoya polynomials of pentachains," MATCH Communications in Mathematical and in Computer Chemistry, vol. 65, pp. 807-819, 2011.

[19] I. Gutman, S. Klavzar, M. Petkovsek, and P. Zigert, "On Hosoya polynomials of benzenoid graphs," MATCH Communications in Mathematical and in Computer Chemistry, vol. 43, pp. 49-66, 2001.

[20] M. Eliasi and B. Taeri, "Hosoya polynomial of zigzag polyhex nanotorus," Journal of the Serbian Chemical Society, vol. 73, no. 3, pp. 311-319, 2008. 
[21] M. Arockiaraj, S. R. J. Kavitha, K. Balasubramanian, and I. Gutman, "Hyper-Wiener and Wiener polarity indices of silicate and oxide frameworks," Journal of Mathematical Chemistry, vol. 56, no. 5, pp. 1493-1510, 2018.

[22] W. Gao, Z. Iqbal, M. Ishaq, R. Sarfraz, M. Aamir, and A. Aslam, "On eccentricity-based topological indices study of a class of porphyrin-cored dendrimers," Biomolecules, vol. 8, no. 3, p. 71, 2018.

[23] Z. Iqbal, M. Ishaq, A. Aslam, and W. Gao, "On eccentricity based topological descriptors of water soluble dendrimers," Zeitschrift für Naturforschung C, vol. 74, 2018.

[24] W. Gao, Z. Iqbal, M. Ishaq, A. Aslam, and R. Sarfraz, "Topological aspects of dendrimers via distance based descriptors," IEEE Access, vol. 7, 2019.

[25] Y. Rao, A. Aslam, M. U. Noor, A. Othman Almatroud, and Z. Shao, "Bond incident degree indices of catacondensed pentagonal systems," Complexity, vol. 2020, Article ID 4935760, 7 pages, 2020. 